\title{
Rapid changes in LLBL thickness
}

\author{
Article
}

Published Version

Hapgood, M. and Lockwood, M. (1995) Rapid changes in LLBL thickness. Geophysical Research Letters, 22 (1). pp. 77-80. ISSN 00948276 doi: https://doi.org/10.1029/94GL02835 Available at https://centaur.reading.ac.uk/38810/

It is advisable to refer to the publisher's version if you intend to cite from the work. See Guidance on citing.

Published version at: http://dx.doi.org/10.1029/94GL02835

To link to this article DOI: http://dx.doi.org/10.1029/94GL02835

All outputs in CentAUR are protected by Intellectual Property Rights law, including copyright law. Copyright and IPR is retained by the creators or other copyright holders. Terms and conditions for use of this material are defined in the End User Agreement.

\section{www.reading.ac.uk/centaur}

\section{CentAUR}

Central Archive at the University of Reading

Reading's research outputs online 


\section{Rapid changes in LLBL thickness}

\author{
Mike Hapgood and Mike Lockwood \\ Rutherford Appleton Laboratory, Chilton, Oxfordshire, UK
}

\begin{abstract}
We employ data from two spacecraft, at the dawn flank of the magnetopause, to investigate fluctuations in the thickness of the low-latitude boundary layer (LLBL). We show the LLBL is considerably thinner shortly after the detection of a flux transfer event than it was during the event. These data are shown to be consistent with the theory of transient increases in the open LLBL thickness caused by a pulse of enhanced reconnection at the magnetopause.
\end{abstract}

\section{Introduction}

Recent observations have used characteristic D-shaped ion velocity distributions to identify a low-latitude boundary layer (LLBL) on open field lines, populated by magnetosheath plasma which has crossed the magnetopause by flowing along newly-opened field lines [Smith and Rodgers, 1991; Gosling et al., 1990; Fuselier et al., 1991]. An analytic theory of time-dependent Petschek reconnection shows how pulses of enhanced reconnection rate at the dayside magnetopause will cause transient increases in the thickness of this open LLBL [Semenov et al., 1991]. This theory supports the concepts proposed by Southwood et al. [1988] and found in 2-dimensional MHD simulations by Scholer [1988]. These papers explain the characteristic set of particle and field signatures at the dayside magnetopause, called flux transfer events (FTEs) [e.g. Farmgia et al., 1988], in terms of transient thickenings of the LLBL, caused by pulses of enhanced reconnection. An alternative model of FTEs has been proposed by Sibeck [1990], whereby the characteristic FTE signatures result from multiple intersections with the LLBL caused by magnetopause surface waves driven by solar-wind pressure pulses. This model predicts a thinning of the LLBL during an FTE [cf. Figure 4 of Sibeck, 1990] but makes no quantitative prediction about the layer thickness either in or away from FTEs.

LLBL thickness is, however, difficult to measure from a single spacecraft because of boundary motions [e.g. Hapgood and Lockwood, 1993]. The two models of FTEs are investigated here using data from two spacecraft of the Active Magnetospheric Particle Tracer Explorer (AMPTE) mission, specifically comparing observations by the United Kingdom Satellite (UKS) and the Ion Release Module (IRM) to monitor variations in LLBL thickness.

\section{Data and Results}

The UKS and IRM spacecraft were launched into similar orbits such that their separation remained $\leq 1000 \mathrm{~km}$ during the period of joint operations (Aug. 1984 to Jan. 1985). Both

\section{Copyright 1995 by the American Geophysical Union.}

Paper number 94GL02835

$0094-8534 / 95 / 94$ GL-02835\$03.00 spacecraft carried a range of space plasma instruments including magnetometers and particle spectrometers - see Bryant et al. [1985] and following papers in the same issue.

We consider an outbound LLBL crossing by both spacecraft around 07:00 UT on 19 Dec 1984. The spacecraft were at 6 hours MLT and a GSM latitude of $+7^{\circ}$. Figure 1 shows $U K S$ and IRM 5 s electron data taken simultaneously during the crossing - as scatter plots of density versus temperature. Both datasets exhibit very similar curved bands but with systematic shifts in temperalure and density. This indicates that the two spacecraft measured the same clectron populations but that there was a marked difference in the calibration - as previously noted by Sibeck [1992]. We can look for similarities and differences between the two datasets using the transition parameter $\tau$ developed at RAL [Bryant and Riggs, 1989; Hapgood and Bryant, 1992] and relate these to the spacecraft separation. This parameter is based on the anticorrelation of electron density and mean energy in the LLBL as demonstrated by the two curved bands of points in Figure 1. (Note that the UKS temperatures in that figure are perpendicular temperatures. This component is preferred for calculating $\tau$ since it gives a slightly better anti-correlation than the parallel component [Hapgood and Bryant, 1992]. However, the IRM temperatures are isotropic averages. T, since $T_{\perp}$ was not available to us - but there is still a clear anti-correlation in Figure 1 because $\mathrm{T}_{\perp}$ and $\mathrm{T}_{\|}$(and hence T) are closely correlated [Hapgood and Bryant, 1992].)

The parameter $\tau$ measures the relative position of data points within each curved band and is normalised such that $\tau=0$ at the magnetosheath end of the band and $\tau=100$ at the magnetospheric end. Thus $\tau$ is a measure of the state of the

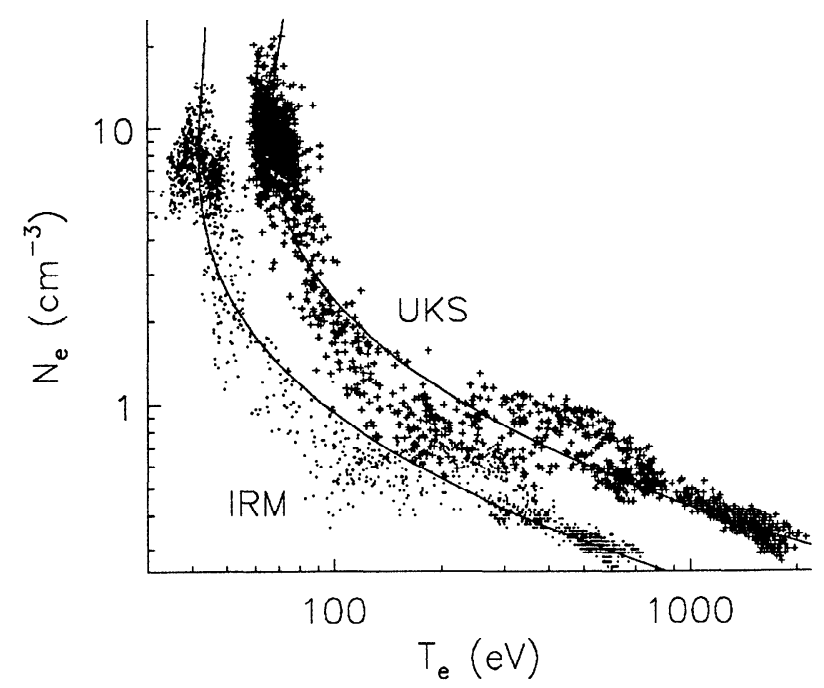

Figure 1. Electron density versus temperature for 05:36 to 08:24UT on 19 December 1984. Crosses indicate UKS data; dots indicate IRM data. The curves are best-fitting cubics for each dataset - as used to calculate the transition parameter. 
LLBL plasma between the magnetosheath and magnetospheric extrema. It is a convenient tool for comparing the $U K S$ and IRM datasets since it is normalised separately within each dataset. Thus we overcome the difference in the calibration between the two datasets.

We also look for differences between the $5 \mathrm{~s}$ average magnetic field data from both spacecraft - presented in boundary normal (LMN) coordinates. The boundary normal $\mathrm{N}$ is taken as the minimum variance direction of the $U K S$ magnetic field data for the period of 05:30-07:30. The $L$ direction is the projection of the dipole axis on the plane perpendicular to $\mathrm{N}$ and $\mathrm{M}$ completes the right-hand set. We also use bulk ion velocity data presented in these boundary normal coordinates, but only from $U K S$ as visual inspection of the two datasets shows that the UKS ion velocities are much less noisy than those from IRM. We attribute this to the fact that the $U K S$ ion instrument, unlike that on IRM, used a high angular resolution mode to measure antisunward ion flows, such as those in the LLBL.

The inter-spacecraft separation during this crossing was about $400 \mathrm{~km}$ - mostly aligned in the $\mathrm{N}$ direction and with UKS more earthward than IRM. The spacecraft velocities with respect to the Earth were about $1.6 \mathrm{~km} \mathrm{~s}^{-1}$ and largely in the $+\mathrm{N}$ direction. Thus if the two spacecraft had been crossing a static boundary we would expect to see a lag of 275 s between any features in the time series recorded by the two spacecraft.

Examination of summary plots of data from UKS and IRM (not shown) reveals that there are many abrupt fluctuations between magnetosheath and magnetosphere between 06:05 and 07:25. Despite this the two datasets are almost identical. All fluctuations are seen in close synchronisation; the lag between the two spacecraft is always $\leq 15 \mathrm{~s}$. This is much less than the nominal lag of 275 s for crossing a static structure. Thus we conclude that the observed fluctuations are nearly all the result of boundary motions. The motions have velocities $\geq 25 \mathrm{~km} \mathrm{~s}^{-1}$ in the boundary normal direction.

To distinguish the differences between the UKS and IRM datasets we must look in detail. We focus on the interval between 06:40 and 07:00 as shown in Figure 2. Here we see two types of fluctuations which are typical of all fluctuations seen during this crossing:

1) there are "slow" events, such as that centred on 06:45, during which $\tau$ changes slowly (timescale $\sim 20$ to 60 s) and there are no significant differences between $U K S$ and IRM measurements of any quantity shown.

2) there are "abrupt" events, such as those after 06:51, during which $\tau$ changes abruptly (timescale $\leq 5 \mathrm{~s}$ ) and there are significant differences between $U K S$ and IRM measurements of $\tau$ and the magnetic field.

The existence of these two types of fluctuations, close together in time, indicates that the scale length of the plasma in the LLBL can change dramatically within minutes. Since the inter-spacecraft separation is orientated normal to the estimated magnetopause direction we can use this scale length as an estimate of the LLBL thickness.

The lack of significant differences between $\tau$ values from $U K S$ and IRM during slow events indicates that the plasma scale length at that time is at least an order of magnitude longer than the spacecraft separation. Thus the scale length, and the LLBL thickness, must have been $>4000 \mathrm{~km}$ during the slow event.

However, during abrupt events there were major and consistent differences between $\tau$ values from $U K S$ and $I R M$. The two time-series of $\tau$ are nested with $I R M$ values systematically more sheathlike than $U K S$ values, which is in agreement with the spacecraft orientation. The large differences between $I R M$ and $U K S$ values (e.g. events around 06:52:50 and 06:58:30) indicate times when the plasma scale length was comparable with the spacecraft separation. Indeed,

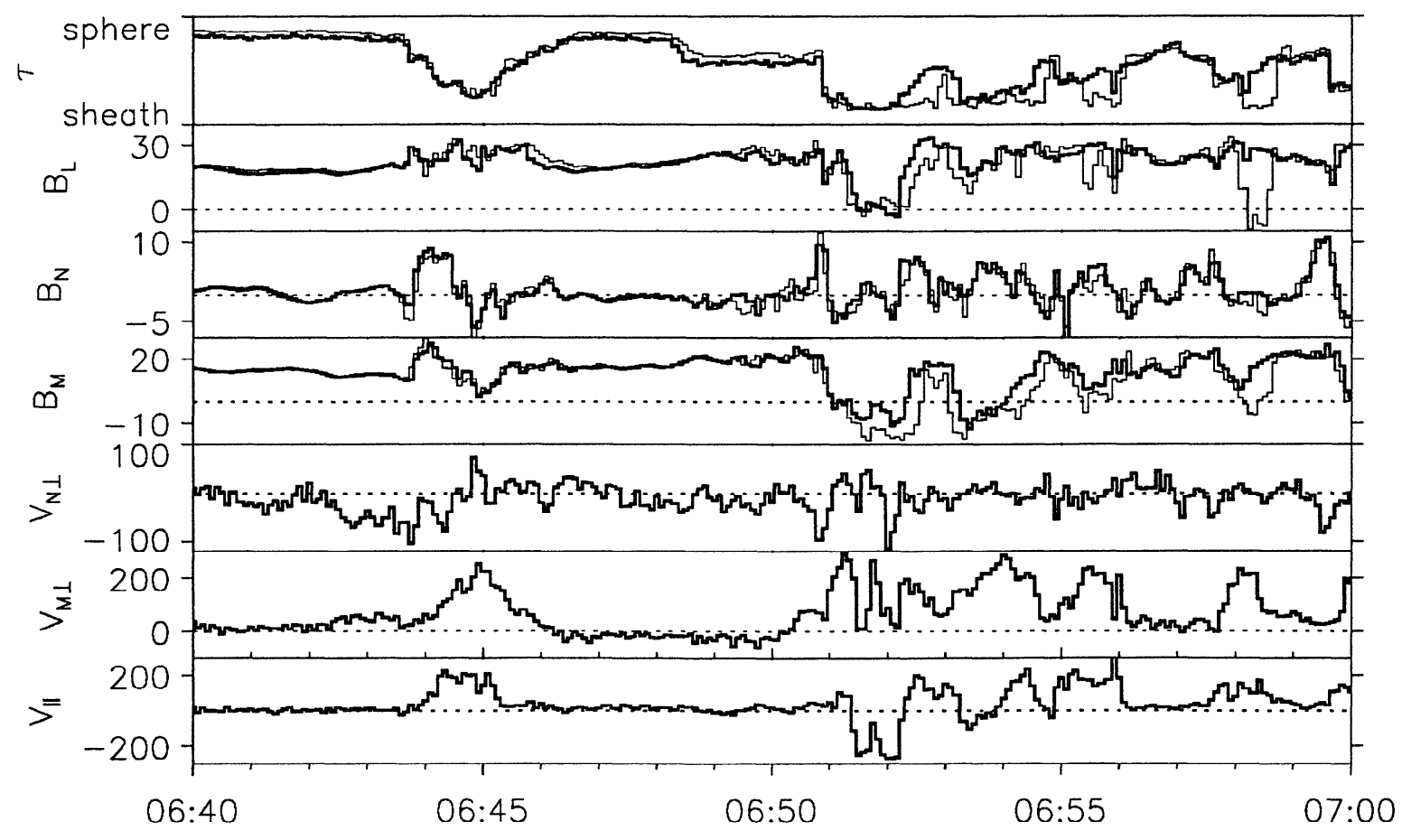

Figure 2. Transition parameter, magnetic field and flow components for 06:40 to 07:00UT. UKS and IRM data are shown by thick and thin curves respectively. 
at these times, the separation vector must have spanned a large fraction of the LLBL. Abrupt changes are also seen in the magnetic field at these times: at 06:52:50 there is a clear nesting signature in $B_{M}$ when both spacecraft see a brief change to magnetospheric fields but $I R M$ sees a shorter event than $U K S$; similarly at 06:58:30IRM sees a short change to magnetosheath fields in $B_{L}$ and $B_{M}$ but this is barely seen on $U K S$. Thus IRM tends to see a magnetic field that is more sheathlike than that seen on UKS, which is again in agreement with the spacecraft orientation. These differences in B confirm our previous conclusion that, at those times, the separation vector must have spanned a large fraction of the LLBL. Thus for these abrupt cases we take the scale length, and the LLBL thickness, to be no more than twice the separation, i.e. $\leq 1000 \mathrm{~km}$.

\section{Discussion}

The data discussed in the previous section show two characteristic behaviours of the LLBL. Between 06:52 and 07:00 the LLBL is relatively thin, to the extent that sometimes most of it lies between the two spacecraft which are just $400 \mathrm{~km}$ apart in the boundary-normal direction. At this time the two spacecraft record nested signatures showing repeated crossings of the LLBL, as invoked by Sibeck [1990] as an explanation of FTE signatures. However, FTE signatures are not observed at this time. The wave-like LLBL transitions instead produce what would be termed " $B_{N}$ activity". Around 06:45 there is an event which is classified as an FTE in the survey of UKS data by South in ood et al. [1986]. At this time, the LLBL is found to be sufficiently thick that there are no differences between the two spacecraft. From this we infer its width to be of order $1 R_{e}$, which is considerably thicker than after 06:51. Figure 3 shows how the 2-D time-dependent reconnection theory of FTEs can explain the observed field and flow features. In this schematic the L component of the field is taken to be zero and the FTE structure is moving along the magnetopause in the M-direction. Note that in this model. the FTE is merely a thickening of the reconnection layer (the open LLBL), around a patch of flux threading the magnetopause, produced by a prior transient pulse of enhanced reconnection rate. The enhanced reconnection increases the boundary-normal magnetic field but proportionally increases the tangential electric field, such that the newly-opened field lines making up the event move along the magnetopause at the same velocity as all other newly-opened field lines [Semenov et al., 1991]. As the resultant thickening of the LLBL passes over the satellite, an FTE signature is observed. The top panel in Figure 3 shows the structure in its own rest frame, in which the satellite, $\mathrm{S}$, moves in the $-\mathrm{M}$ direction. The lower panels show the variation of the transition parameter, field and velocity components as observed by the satellite. In an open LLBL, the plasma parameters vary as a function of the fieldaligned distance from the magnetopause because of ion timeof-flight effects. This means that $\tau$ will decrease as the fieldaligned distance from $S$ to the magnetopause decreases [T.G. Onsager, private communication, 1994]. The transition parameter is a minimum at the centre of the event where $S$ observes field lines closest to where they cross the magnetopause. At these points plasma is seen streaming along the newly-opened field lines into the magnetosphere $\left(\mathrm{V}_{||}>0\right)$. The other field and flow components in the MN

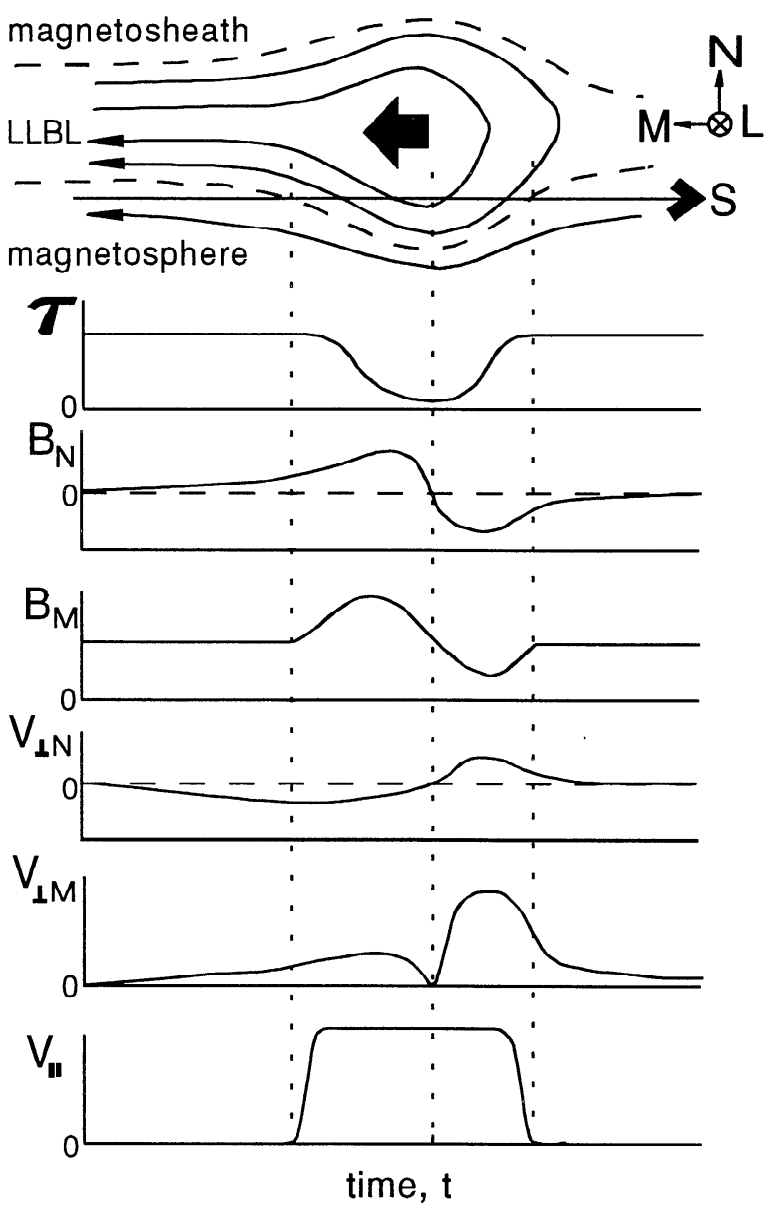

Figure 3. 2-D model of an FTE propagating along the magnetopause from the conceptual modelling of Southwood et al. [1988], simulations by Scholer [1988] and analytic theory by Semenov et al. [1991]. The lower pancls are schematic curves showing its signature in transition parameter, magnetic field and velocity components.

plane of Figure 3 arise from the field topology and the event motion in the $\mathrm{M}$ direction. As the leading part of the event passes over $\mathrm{S}$, it observed an outward $\mathrm{B}_{\mathrm{N}}$, as noted by Southwood et al. [1988] and $\mathrm{B}_{\mathrm{M}}$ is increased as the field is compressed. The motion in the $\mathrm{M}$ direction gives fieldperpendicular flow with components $\mathrm{V}_{\perp \mathrm{N}}<0$ and $\mathrm{V}_{\perp \mathrm{M}}>0$. At the event centre $B_{N}$ goes to zero and the event velocity coincides with the field direction (for this 2-D structure with $B_{L}=0$ ). Subsequently $B_{N}$ is negative and $B_{M}$ falls as field lines thread the magnetopause. The event motion results in field-perpendicular flow components $\mathrm{V}_{\perp \mathrm{N}}>0$ and $\mathrm{V}_{\perp \mathrm{M}}>0$. This FTE signature is somewhat unusual in that the $M$ component shows a bipolar variation (about a positive average value), roughly in phase with that in $\mathrm{B}_{\mathrm{N}}$. This is explained in Figure 3 because the satellite is sufficiently close to the magnetopause after the passage of the FTE to see the low-field region in its immediate wake, caused by magnetic flux penetrating the boundary within the FTE.

Comparison of Figures 2 and 3 shows that this model reproduces all the observed features well, with a single exception. That is, when $B_{N}$ is zero, the $M$ component of the field perpendicular flow is predicted to go to zero, whereas, Figure 2 reveals a small dip, but not down to zero. This is easily explained by adding the observed positive L compo- 
nent to the model field (giving an internal twist to the FTE field) which generates a positive $V_{\perp M}$ and a negative $V_{\perp L}$ at this point, as is observed. We conclude that the model of Semenov et al. [1991], Scholer [1988] and Southwood et al. [1988] does agree well with the ion flow and magnetic field characteristics in this FTE, as well as with the electron data. as characterised by the transition parameter.

The interesting point about the FTE model depicted in Figure 3 is that it predicts a transient thickening of the LLBL. Independent evidence for this comes from comparison of the electron data from the two spacecraft. Figure 2 shows that at any one time during the intersection with this event. there are no significant differences between the transition parameter values, derived simultaneously from the two spacecraft. There is no nesting, as observed just 7 min. later. Hence this study of the thickness of the LLBL supports the concept that the FTE is a transient thickening of the open LLBL. No estimates of the boundary thickness in the 15 minutes prior to the event are possible as the satellite was in the magnetosphere for all of that period. The data only reveal a rapid decrease in the LLBL thickness immediately after the FTE. The FTE model predicts both this and a rapid thickening of the LLBL at the start of the event. This could have been present but the spacecraft were in the wrong location to detect it.

The pressure pulse model, on the other hand, predicts a thinning of the LLBL during the event as the surface wave passes over spacecraft which otherwise were within the magnetosphere [Figure 4 of Sibeck, 1990]. It also predicts nested plasma and field variations. Neither of these were observed in the FTE studied here, but the nested signatures observed after 06:52 were consistent with surface waves. However, no FTE signatures were then detected.

The inferred event motion close to the $\mathrm{M}$ direction is also interesting: were there to have been a significant $\mathrm{L}$ component to this motion, then events produced by reconnection at the low-latitude dayside magnetopause would not he carried to the satellites which were at the dawn flank of the magnetopause at low latitudes. Hence the fact that the event motion appears to be well-aligned with the $\mathrm{M}$ direction supports the idea that this is an FTE structure produced by a reconnection pulse at an $\mathrm{X}$-line at low latitudes on the dayside magnetopause.

Acknowledgements. We acknowledge the use of data from other instruments on the AMPTE spacecraft: D. Southwood (Imperial College London) and A. Johnstone (MSSL) for UKS magnetometer and ion data, and $\mathrm{H}$. Lühr (TU Braunschweig) and G. Paschmann (MPE Garching) for IRM magnetometer and electron data respectively. The UK Geophysical Data Facility provided an on-line archive of data from AMPTE-UKS and AMPTE-IRM.

\section{References}

Bryant, D.A., Krimigis, S.M. and Haerdendel, G., Outline of the AMPTE Mission, IEEE Trans. Geosci. Remote Sensing, GE23, 177-181, 1985.

Bryant, D.A. and S. Riggs, At the edge of the Earth's magnetosphere: a survey by AMPTE-UKS. Phil. Trans. R. Soc. Lond. A328, 43-56, 1989.

Farrugia, C. J., R. P. Rijnbeek, M. A. Saunders, D. J. Southwood, D. J. Rodgers, M. F. Smith, C. P. Chaloner, D. S. Hall, P. J. Christiansen, and L. J. C. Woolliscroft, A multi-instrument study of flux transfer event structure, $J$. Geophys. Res., 93, 14465-14477, 1988.

Fuselier, S.A., D.M. Klumpar, and E.G. Shelley, Ion reflection and transmission during reconnection at the earth's subsolar magnetopause, Geophys. Res. Lett., 18, 139-142, 1991.

Gosling, J.T., M.F. Thomsen, S.J. Bame, R.C. Elphic, and C.T. Russell, Cold ion beams in the low latitude boundary layer during accelerated flow events, Geophys. Res. Lett., 17, 2245-2248, 1990.

Hapgood, M.A. and D.A. Bryant, Exploring the magnetospheric boundary layer. Planet. Space Sci., 40, 1431-1459, 1992

Hapgood, M.A. and M. Lockwood, On the voltage and distance across the low latitude boundary layer, Geophys. Res. Lett., 20, 145-148, 1993.

Scholer, M., Magnetic flux transfer at the magnetopause based on single X-line bursty reconnection, Geophys. Res. Lett., 15, 291-294, 1988.

Semenov, V.S., I.V. Kubyshkin, H.K. Biernat, M.F. Heyn, R.P. Rijnbeek, B.P. Besser, and C.J. Farrugia, Flux transfer events interpreted in terms of a generalized model for Petschek-type reconnection, Adv. Space Res., 11, (9)25-(9)28, 1991.

Sibeck, D.G., A model for the transient magnetospheric response to sudden solar-wind dynamic pressure variations, J. Geophys. Res., 95, 3775-3771, 1990 ).

Sibeck, D.G., Transient events in the outer magnetosphere: boundary waves or flux transfer events?. J. Geophys. Res., 97, 4009-4026, 1992.

Smith, M.F. and D.J. Rodgers, Ion distributions at the dayside magnetopause, I. Geophys. Res., 96, 11,617-11.624, 1991.

Southwood, D.J., M.A. Saunders, M.W. Dunlop, W.A.C. Mier-Jedrzejowicz and R.P. Rijnbeek, A survey of flux transfer events recorded by the UKS spacecraft magnetometer, Planet. Space Sci. 34, 1349-1359, 1986.

Southwood, D. J., C. J. Farrugia, and M. A. Saunders. What are flux transfer events?, Planet. Space Sci., 36, 503-508, 1988.

Mike Hapgond \& Mike Inckwond, Rutherford Appleton Laboratory, Chilton, Didcot, Oxfordshire, OX11 OQX, United Kingdom. (e-mail: Internet. M.Hapgood@rl.ac.uk)

(Received: August 31, 1994; accepted: September 29, 1994.) 